\title{
Intercambiabilidad de medicamentos de origen biológico (biofármacos): Consideraciones acerca de la aprobación de formulaciones biosimilares (biogenéricos) en Chile
}

\author{
Iván Saavedra $\mathbf{S}^{\mathbf{l a}}$, Luis Q uiñones $\mathbf{S}^{\mathbf{2 b}}$. \\ Interchangeability of biological drugs: \\ Considerations about the approval of \\ biogeneric formulations in Chile
}

Once drug patents expire, the health authorities can approve the registry of similar products. They must request to the manufacturer, the bibliographic background of the original product and the analytical results that certify drug quality. An inspection of the premises of the manufacturer is also required. The main goal of this approval is to decrease cost, considering that the original product is usually more expensive. This is a current situation due to the imminent expiration of the patents of many biopharmaceutical products. Therefore, in Chile, the Public Health (ISP) and the Ministry of Health should consider that for this kind of products, until now, there are no interchangeable generic drugs, and that the similar drugs that are offered have a different chemical composition, since they have been manufactured through different processes. In the case of biological drugs (e.g. erythropoietir, somatotropin, heparin) the quality and homogeneity depend from the manufacture process. Its complete composition can not be absolutely elucidated; therefore small impurities or conformational variants can elicit an altered immune response or unexpected adverse reactions. This indicates that the approval of a biogeneric drug requires in addition to pharmacokinetic studies, preclinical and clinical analytical studies such as physicochemical assays, biological and immunological test. This issues have been established by WHO and have been incorporated for the main drug registry entities all over the world (FDA, EMEA, ANVISA) to approve biogeneric products (Rev Méd Chile 2006; 134: 1583-88).

(Key words: Drug industry; Erythropoietin; Heparin, low-molecular-weight; Human growth hormone)

Recibido el 2 de noviembre, 2005. Aceptado el 19 de junio, 2006.

${ }^{1}$ Laboratorio de Farmacocinética y Biodisponibilidad, IFT, Programa de Farmacología Molecular y Clínica, ICBM, Facultad de Medicina, Universidad de Chile. ${ }^{2}$ Laboratorio de Carcinogénesis Química y Farmacogenética, IFT, Programa de Farmacología Molecular y Clínica, ICBM, Facultad de Medicina, Universidad de Chile.

a Químico Farmacéutico.

b Bioquímico, PhD.

Correspondencia a: Iván Saavedra Saavedra. Instituto de Investigaciones Farmacológicas y Toxicológicas (IFT). Programa de Farmacología Molecular y Clínica, ICBM, Facultad de Medicina, Universidad de Chile. Casilla 70111, Santiago 7, Chile. Teléfonos: 56-2-6817756-56-2-9786414.

E mail: isaavedr@med.uchile.cl 
T os países incorporados a la Organización Munـdial del Comercio tienen la obligación de respetar las patentes comerciales de inventos, como es el caso de los medicamentos innovadores. Esta obligación se acrecienta por la existencia de tratados comerciales con aquellos países desde donde proceden los productos patentados. Así, la salida al mercado de productos similares o copias está vedada, hasta que la licencia otorgada al innovador termine ${ }^{1}$.

Una vez caducadas las patentes, las autoridades sanitarias quedan en libertad de permitir el registro de similares o copias del original y aprobar su salida al mercado, exigiéndole al fabricante antecedentes bibliográficos acerca de la droga original y resultados analíticos que avalen la calidad del producto; además, inspeccionan sus instalaciones y revisan el etiquetado ${ }^{2}$. Esta aprobación está concebida para bajar los costos de los tratamientos debido a que en la mayoría de los casos, el producto original tiene precios mayores que los medicamentos similares. Esta es la situación que actualmente se está viviendo en el mundo y particularmente en nuestro país, debido a la inminente expiración de las primeras patentes de medicamentos de origen biológico.

En aquellos países que han suscrito los acuerdos de la OMS referentes a la fabricación de genéricos para la intencambiabilidad, las autoridades exigirán, además de los antecedentes bibliográficos, pruebas de biodisponibilidad para demostrar bioequivalencia con el original o bien, si las circunstancias lo amenitan, estudios clínicos, farmacodinámicos o pruebas fisicoquímicas, dependiendo de la naturaleza de la droga y de la forma farmacéutica que tendrán los futuros genéricos. Con ello se persigue, además de bajar los costos del tratamiento, asegurar al médico y al paciente que el genérico en estudio cuenta con la misma seguridad y eficacia demostrada mediante estudios clínicos multicéntricos por el medicamento original $^{3}$. Al respecto, en 1984 el congreso norteamericano estipuló que los genéricos bioequivalentes producen similar seguridad y eficacia que el innovador y tienen beneficio económico 4 .

Existen en la actualidad 6 criterios básicos de intercambiabilidad ${ }^{5}$ :

1. Equivalencia farmacológica

2. Evidencia clínica que sostenga la intercambiabilidad terapéutica.

3. Estudios de costo/disponibilidad.

4. Completo proceso de evaluación (ej. Comités terapéuticos y de Farmacia).

5. Monitoreo regular de egresos de pacientes.
6. Análisis de las respuestas variables.

El medicamento original se toma como estándar de comparación debido a que fue registrado con numerosas pruebas científicas y técnicas, fisicoquímicas, clínicas, farmacodinámicas, farmacocinéticas y farmacéuticas que avalaron calidad, seguridad y eficacia.

Las agencias de aprobación de medicamentos más desarrolladas del mundo, la FDA (Food and Drug Administration), la EMEA (European Agency for the Evaluation of Medicinal Products) y la OMS, han emitido numerosas declaraciones y guías de orientación para la aprobación de la intercambiabilidad terapéutica, tanto para sus propios países como para los países con menor desarrollo farmacológico $^{6}$. Si se considera que la FDA comenzó con esta política en el año 1984 y por ejemplo, España aprobó la ley del medicamento sólo en 1992, podemos concluir que aún quedan situaciones que es necesario aclarar, discutir o proponer.

Un ejemplo claro de esta ambigüedad es la escasa literatura y guías relacionadas con la aprobación de productos similares y genéricos que poseen principios activos de origen biológico (biofármacos). Estos medicamentos, a diferencia de las drogas que tienen un origen en la síntesis química (las que se pueden obtener con una alta pureza), muchas veces son mezclas de principios activos o de macromoléculas con actividad biológica, cuya composición dependerá de muchas variables en los complicados procesos de elaboración y cuya composición específica es muy difícil de definir con las tecnologías actuales. Debido a esto es que existen numerosos argumentos que hacen la aprobación de este tipo de medicamentos biosimilares mucho más complicada que para los equivalentes genénicos de fármacos convencionales.

En el presente trabajo se discutirá acerca de los fundamentos científicos para la identificación de medicamentos biosimilares, su aprobación y las implicancias en la salud pública.

\section{CONCEPTOS FUNDAMENTALES DE LOS BIOFÁRMACOS}

Los medicamentos de origen biológico son productos medicinales biotecnológicos que poseen una complejidad intrínseca y que se definen como sustancias que son producidas desde una fuente biológica o extraídas de ella y que requieren para su caracterización y determinación de calidad, de una combinación de pruebas fisicoquímicas y biológicas, 
conjuntamente con el proceso y control de la producción. Ejemplos de biofármacos son las citoquinas, hormonas, anticuerpos y vacunas?

En muchos casos, estos biofámacos son proteínas recombinantes cuya calidad y homogeneidad dependen del proceso de producción por la célula hospedera y de los procesos de purificación y formulación, por lo que para su aprobación como biosimilar se requenirá además de los estudios farmacocinéticos, de pruebas adicionales analíticas, preclínicas y clínicas. Chinno y Mire-Sluis ${ }^{8}$, recientemente, han revisado las diferentes pruebas de caracterización y comparabilidad de biofármacos, las que incluyen pruebas fisicoquímicas, biológicas, inmunogénicas y farmacoci- néticas, señalando la instrumentación requerida y las implicancias de las divergencias analíticas. La Tabla 1 muestra un listado de medicamentos de origen biológico cuya patente ya expiró o se encuentra pronta a expirar, razón por la cual se transforman en blancos de fabricación de biosimilares.

La legislación actual en la comunidad europea ya contempla la posibilidad de presentar solicitudes para este tipo de fármacos $\mathrm{y}$, de hecho, ya ha habido presentaciones de medicamentos biosimilares a través de EMEA. Para ello, la documentación que la compañía debe presentar es la misma que en el caso de un fármaco nuevo, con pequeñas variantes de acuerdo al producto; posteriormente, se observa

Tabla 1. Algunos medicamentos de origen biológico con patente expirada o cercana a la expiración (2005-2007).

\begin{tabular}{|c|c|c|c|}
\hline $\begin{array}{l}\text { Producto } \\
\text { (marca registrada) }\end{array}$ & Principio Activo & $\begin{array}{l}\text { Propietario de la Patente } \\
\text { (solicitante) }\end{array}$ & $\begin{array}{l}\text { Patentes asociadas al } \\
\text { producto y fecha de } \\
\text { publicación internacional }\end{array}$ \\
\hline Nunotropin & Somatotropina & Genentech & $\begin{array}{l}\text { CA2345497 }(28 / 04 / 1990) \\
\text { CA1267099 }(27 / 03 / 1990) \\
\text { AU627174B }(20 / 08 / 1992) \\
\text { US5096885 }(17 / 03 / 1992)\end{array}$ \\
\hline TNKase & Tenecteplasa TNK-tPA & & CA1271149 (03/07/1990) \\
\hline Activase & TPA & & US5011795 (30/04/1991) \\
\hline Alteplase & $\mathrm{tPA}$ & & HU206520 (30/11/1992) \\
\hline Abbokinase & Eudurasa uroquinasa & Abbott & $\begin{array}{l}\text { W09004024 (19/04/1990) } \\
\text { EP0379545 (01/08/1990) } \\
\text { EP0414828 (06/03/1991) }\end{array}$ \\
\hline Humulin & Insulina recombinante & Eli Lilly & $\begin{array}{l}\text { AU595934B (12/04/1990) } \\
\text { AU5483390 (15/11/1990) }\end{array}$ \\
\hline Humatrope & Somatotropina & & $\begin{array}{l}\text { AU594047B (01/03/1990) } \\
\text { NZ228313 (27/11/1990) } \\
\text { AU614551B (15/09/1991) } \\
\text { US5068317 (26/11/1991) }\end{array}$ \\
\hline Ceredase & Alglucerasa & Genzyme & $\begin{array}{l}\text { CA2339888 }(23 / 06 / 1990) \\
\text { CA2006709 }(23 / 06 / 1990) \\
\text { W09007573 }(12 / 07 / 1990) \\
\text { W09213067 }(06 / 08 / 1992)\end{array}$ \\
\hline Cerezyme & Imiglucerasa & & US5236838 (17/08/1993) \\
\hline Streptase & Streptoquinasa & Astrazeneca & $\begin{array}{l}\text { GB2226495 }(04 / 07 / 1990) \\
\text { CA2000850 }(19 / 04 / 1990) \\
\text { AU592862B }(25 / 01 / 1990)\end{array}$ \\
\hline Intron A & INFə2ß & Biogen/Roche & CS9004328 (16/12/1992) \\
\hline Serostim & Somatotropina & Serono & US4939124 (03/07/2005) \\
\hline Epogen & Eritropoyetina & Amgen & AU600650B (23/08/1990) \\
\hline Procrit & Eritropoyetina & & NZ222432 (26/07/1990) \\
\hline Eprex & Eritropoyetina & & AU5750490 (04/10/1990) \\
\hline Neupogen & Filgrastrim G-CSF & & CA2311684 (22/06/1990) \\
\hline Proleukin & IL-2 & Chiron/Cetus & $\begin{array}{l}\text { EP0357645 (14/03/1990) } \\
\text { EP0368857 (23/05/1990) } \\
\text { EP0470586 (12/02/1992) }\end{array}$ \\
\hline
\end{tabular}


cuán similar es él con respecto al producto que se ha tomado como referencia ${ }^{6}$. La EMEA ha creado recientemente el Biosimilar Working Party, que es un grupo que está trabajando en guías específicas de productos sobre requerimientos de estudios clínicos para los fármacos biosimilares. Actualmente, hay borradores definidos para insulina, hormona de crecimiento, enitropoyetina y factor estimulador de colonias de granulocitos, y se encuentra en preparación la de interferón alfa.

En mayo de 2004, el parlamento europeo realizó una serie de recomendaciones para la aprobación de compuestos biogenéricos ${ }^{9}$. Estas recomendaciones fueron acogidas por la EMEA para generar guías de aprobación de estos biosimilares. Sin embargo, existe incerteza respecto de las pruebas y de la documentación clínica necesaria requeridas para esta aprobación, además de una falta de uniformidad de criterios entre los diferentes grupos regulatorios. Las normas regulatorias para la evaluación de bioequivalencia en biofármacos, establecidas por la $\mathrm{EMEA}^{10}$, son dirigidas a argumentos clínicos y de la calidad de los biofármacos y establecen, en términos generales, que el fabricante debe demostrar perfiles de calidad, seguridad y eficacia similares entre el producto nuevo o modificado y el innovador. En este aspecto, se debe considerar la complejidad de las estructuras moleculares, los cambios incorporados en el proceso de manufactura y las implicancias de ellos en el funcionamiento seguro del fármaco. Se señala además, que en ciertos casos, una equivalencia satisfactoria no puede ser demostrada y, por tanto, se requiere de pruebas preclínicas completas y datos clínicos compilados satisfactorios ${ }^{6}$. De este modo, pruebas simples, como los ensayos de validación o las pruebas farmacocinéticas de bioequivalencia no serán suficientes, a menos que los métodos analíticos sean capaces de caractenzar la complejidad de los biofármacos y establecer una comparabilidad predictiva. Al respecto, existe una serie de problemas asociados con los estudios de bioequivalencia en biosimilares, por ejemplo, los biofármacos son un gran número de moléculas complejas y heterogéneas con también complejos mecanismos de acción. La relación entre la farmacodinamia y el efecto clínico de los biofármacos no es clara hoy en día, los biofármacos tienen múltiples blancos de acción, los ensayos para biofármacos son pocos, a menudo ambiguos y difíciles de llevar a cabo y, finalmente, los marcadores de eficacia de estos biofármacos no son muy claros.
La inmunogenicidad es una de las mayores preocupaciones en fámacos biosimilares, dado que especies alteradas conformacionalmente 0 pequeñas impurezas del proceso pueden conducir a una respuesta inmune que puede producir un efecto inhibitono en la acción del medicamento o una respuesta alérgica. Un caso relevante al respecto ha sido el de Eprex, que comesponde a eritropoyetina $\alpha$, la que ha sido asociado a una aplasia de glóbulos rojos junto con producción de anticuerpos neutralizantes de enitropoyetina, lo que a su vez parece estar relacionado con los cambios de formulación ${ }^{11,12}$. Lamentablemente, hoy en día no existe un modelo animal validado para evaluar la inmunogenicidad de proteínas, motivo por el cual, la segunidad inmunológica debe ser probada en estudios clínicos y vigilancia posmercado.

Un ejemplo relevante lo constituyen las heparinas de bajo peso molecular (HBPMs) las cuales exhiben diferentes cualidades fisicoquímicas (pesos moleculares y estructuras) que son determinadas por el proceso de manufactura que se utiliza ${ }^{5}$. Por ejemplo, la enoxaparina es preparada por un proceso de bencilación seguido de una hidrólisis en medio alcalino de la heparina no fraccionada (HNF). Este procedimiento, también patentado, da como resultado un doble enlace en el carbono terminal del último monosacárido no reductor del polisacárido; además de la presencia de una estructura bicíclica única denominada 1,6 anhidro manosa en el monosacárido terminal reductor del polisacárido. Esta estructura se puede presentar en forma variable de $5 \%$ a $50 \%$ en el producto ramificado, dependiendo del proceso de fabricación ${ }^{5}$, lo que influencia su actividad anti-Xa y su afinidad hacia ATIII. Otras HBPMs, tales como la dalteparina, nadroparina, tinzaparina y parnaparina, obtenidas con procedimientos de fabricación diferentes, exhiben otras estructuras específicas que serían la causa de sus propios perfiles bioquímicos y farmacológicos. $\mathrm{Si}$ bien es cierto que la actividad anticoagulante es la misma, la diferencia radica en la farmacocinética y actividades biológicas, tales como la interacción con factores de crecimiento celular, con los componentes de la sangre y con las células vasculares. Esta es la razón por la cual las principales agencias de registro de medicamentos como FDA y EMEA, además de la OMS, han tenido la preocupación de no aprobar genéricos intercambiables en estas HBPMs y consideran que cada una de ellas es un agente farmacológico distinto ${ }^{13-15}$. 
Otro caso particular lo constituyen los anticuerpos monoclonales, en los que su variabilidad puede estar dada por sus niveles de glucosilación, formación de puentes disulfuros alternativos, desamidaciones y formación de residuos isoaspartilos, oxidaciones de metionina, ciclación de residuos de glutamina amino-terminales e hidrólisis parcial de residuos de lisina en el carboxilo-terminal ${ }^{7}$.

\section{ASPECTOS RELACIONADOS A LA MANUFACTURA}

Los procesos de manufactura de biofármacos son, en general, muy complejos e involucran numerosos pasos de producción en cascada, razón por la cual el producto final puede estar influenciado en cualquiera de estos pasos. Por ejemplo, se ha descrito 9 pasos generales para la producción de proteínas obtenidas desde cultivos de mamíferos y un total de 244 pasos analíticos para interferón $\alpha-2 \beta$ (Intron-A). Estos diversos pasos, que son particularmente pertinentes a gran escala, afectan diversas características del producto final, como son la estabilidad al almacenamiento y la composición de las impurezas que son elementos que influencian su bioequivalencia. Por lo tanto, para asegurar la calidad del medicamento, se debe hacer un monitoreo riguroso de los parámetros del proceso, el cual es mucho más estricto que para un medicamento convencional.

\section{DisCUSIÓN}

Histónicamente, los productos biológicos han sido mezclas complejas de diferentes especies moleculares, difíciles de caracterizar como entidades individuales, su heterogeneidad intrínseca tiene la propiedad de afectar la eficacia terapéutica. Distinto a los medicamentos convencionales, los biofármacos no pueden ser completamente caracterizados por métodos fisicoquímicos o bioensayos. Por otro lado, la relación entre las propiedades fisicoquímicas y su actividad biológica no está muy bien definida, por ejemplo, diferencias en el grado de glucosilación entre eritropoyetinas $\alpha$ y $\beta$ parecen ser, al menos parcialmente, responsables de las diferencias observadas en su actividad biológica ${ }^{16}$. Por lo tanto, los medicamentos biosimilares no son comparables per se a los innovadores desde el punto de vista de la seguridad y eficacia. Otra manera de ejemplificar esta no intercambiabilidad absoluta, está dada por la somatotropina u hormona de crecimiento humana, que es manufacturada por 6 compañías en el mundo. Aunque todas ellas poseen 191 aminoácidos, peso molecular de 22 Kda y no son glucosiladas, se metabolizan a diferentes velocidades, con vidas medias que varían entre 1,75 horas y 7 a 10 horas $^{17}$, lo que hace que no comespondan a medicamentos bioequivalentes. Lo mismo ocurre para las eritropoyetinas manufacturadas en Asia, India y Sudamérica ${ }^{18}$.

Al contrario de los medicamentos convencionales que se generan mediante procesos de extracción con reactivos químicamente fuertes (solventes orgánicos, ácidos y bases) que eliminan cualquier contaminación del producto original, los biofármacos, cuyo material de partida es generalmente un cultivo celular de miles de litros, con reactivos de origen biológico que constituyen una posible fuente de contaminación viral, requieren un control más estricto en cuanto a los aspectos de seguridad viral o microbiológica. Al ser productos proteicos no pueden someterse a tratamientos tan estrictos como los de síntesis química y los procesos de purificación consisten en filtraciones y cromatografías para mantener la integridad de la proteína.

En conclusión, los organismos internacionales más importantes, tales como EMEA, FDA y OMS están de acuerdo en que para asegurar que la eficacia y seguridad de un medicamento biosimilar son iguales a las del innovador, es necesario demostrar más que una similitud esencial como ocurre con los medicamentos convencionales, y realizar una gran cantidad de estudios que incluyen, análisis cualitativos que especifiquen analogía en cuanto a composición, desarrollar estudios preclínicos que incluyan estudios farmacocinéticos y farmacodinámicos comparativos en humanos, estudios en modelos animales para dosificación, ruta de administración, toxicología, inmunogenicidad, carcinogenicidad, genotoxicidad, teratogénesis y tolerancia local. Es evidente entonces, que el desarrollo de fármacos biosimilares es mucho más complicado que el de los fármacos convencionales y que la equivalencia fisicoquímica, biológica, farmacológica, toxicológica y de dosis debe ser analizada.

En nuestro país, el Instituto de Salud Pública (ISP), encargado del registro de fármacos, no posee política alguna en este aspecto, tan sólo existe una discusión al respecto en el Departamento de Control 
Nacional. Actualmente se considera producto nuevo a las vacunas y algunos anticuerpos monoclonales, pero en general no se establece mayores diferencias entre los medicamentos de origen puramente químico y los de origen biológico.

Por lo tanto, el ISP, en conjunto con el Ministerio de Salud y las diversas instituciones que deciden compras para los servicios hospitalarios, deben considerar que en este tipo de productos no hay, hasta la fecha, en el mundo, genénicos intercambiables, dado que sus composiciones en rigor no han sido totalmente dilucidadas y que los biosimilares que se ofrecen, tienen diferente composición química que el

\section{REFERENCIAS}

1. Tratado de Cooperacion en Materia de Patentes (PCT) (19/06/1970), elaborado en Washington, enmendado el 02/10/1979 y modificado el 03/02/ 1984, y su reglamento (BOE 26/11/1986).

2. MINSAL Reglamento del sistema nacional de registro de productos farmacéuticos. Decreto Supremo 1876, 1995.

3. OMS. Guideline. Multisource (generic) pharmaceutical products: guidelines on registration requirements to stablish interchangeability. WHO Technical Reports Series 863: 114-55.

4. FDA. Drug Price Competition and Patent Term Restoration Act of 1984, Publ. L. No98-417, 98 Stat. 1585.

5. Fareed J, Leong W, Hoppesteadt DA. Generic lowmolecular-weight heparins: some practical considerations. Semin Thromb Hemost 2004; 30 (6): 703-13.

6. Committee for the Propietary Medicinal Products. Guideline on comparability of medicinal products containing biotechnology-derived proteins as active substance. Quality issues. Evaluation of medicine for human use. The European Agency for the Evaluation of Medicinal Products, London; 2003.

7. SCHELLEKENS H. Follow-on biologics: challenges of the enext generation». Nephrol Dial Transplant 2005; 20(4): iv31-iv36.

8. Chirino AJ, Mire-Sluis A. Characterizating biological products and assessing comparability following manufacturing changes. Nature Biotechnol 2004; 22: 1383-91.

9. European Pardiament and Council. Regulation (EC) № 726/2004 of the European Parliament. Europa L136 2004; 47. innovador, puesto que han sido preparados en forma diferente y que por lo tanto, se debe decidir la intercambiabilidad de éstos sobre la base de estudios clínicos y no clínicos apropiados que demuestren seguridad y eficacia. Los requerimientos para demostrar bioequivalencia de fármacos de onigen biológico deben entonces estudiarse caso a caso por expertos y basado en pautas científicas pertinentes. Sugerimos sin embargo, basado en las recomendaciones de FDA, EMEA, OMS y ANVISA, que todo medicamento de onigen biológico que ingrese a nuestro país sea considerado como producto nuevo, de modo de evitar los riesgos potenciales que estos implican.

10. Committee for the Propietary Medicinal Products. Guideline on comparability of medicinal products containing biotechnology-denived proteins as active substance. Non-clinical and clinical issues. Evaluation of medicine for human use. The European Agency for the Evaluation of Medicinal Products, London; 2003.

11. SCHELEKENS H. Relationship between biopharmaceutical immunogenicity of epoetin alfa and pure red cell aplasia. Curr Med Res Opin 2003; 19: 433-4.

12. HASELBECK A. Epoetins: differences and their relevance to immunogenicity. Curr Med Res Opin 2003; 19: 430-2.

13. Nightingale SL. From the food and drug administration. JAMA 1993; 270: 1672.

14. HirSh J, Warkentin TE, Raschke R. Heparin and low molecular weight heparin: mechanism of action, pahramcokinetics, dosing considerations, monitoring, afficacy and safety. Chest 1998; 114(5): 489s-510s.

15. FAREED J, Fu K, Yang LH. Pharmacokinetics of low molecular weight heparins in animal models. Semin Thromb Hemost 1999; 25(3): 51-5.

16. Storring PL, Tiplady RJ, Gaines Das Re et al. Epoetin alfa and beta differ in their eritropietin isoform compositions and biological properties. Br J Haematol 1998; 100: 79-89.

17. RAINES LJ. Bad medicine: why the generic drug regulatory paradigm is inapplicable to biotechnology products. Biolaw bus 2002; 5: 6-13.

18. SCHELEKENS H. Biosimilar epoetins: how similar are they. Eur J Hosp Pharm 2004; 3: 43-7.

\section{Agradecimientos}

Los autores de este trabajo agradecen la gentileza del Dr. Jorge Soto Labbé por su revisión crítica de este manuscrito. 